\title{
Proactive interference of a sequence of tones in a two-tone pitch comparison task
}

\author{
TIMO RUUSUVIRTA \\ University of Jyväskylä, Jyväskylä, Finland
}

\begin{abstract}
Subjects compared pitches of a standard tone and a comparison tone separated by $1,300-3,000 \mathrm{msec}$ and responded according to whether the comparison tone sounded higher or lower in pitch than the standard tone. Three interfering tones at $300-\mathrm{msec}$ intervals were presented before each pair of tones. Their pitch range varied, being either below or above the pitch of the standard tone; in some of the trials, their pitches were identical to the pitch of the standard tone (no interference). The highest error rate in performance was found when the interfering tones and the comparison tone deviated in the same direction in pitch from the standard tone. In turn, their deviations in the opposite directions resulted in the lowest error rate. This effect was not found to be dependent on whether the interfering tones were randomly ordered or monotonically ordered, together with the standard tone, into melodically ascending/descending sequences. An intermediate error rate in performance was found when the interfering tones and the standard tone were identical. The results support earlier hypotheses, presented in the context of retroactive interference, by demonstrating proactive interference of a tone sequence at the level of representations of individual tones.
\end{abstract}

When pitch comparisons are made between two tones (standard and comparison tones, respectively) separated by a sequence of interfering tones, the error rate in performance varies as a function of the pitch relationship between the stimuli. Performance improves when the pitch of the standard tone occurs in the sequence and deteriorates when the pitch of the comparison tone occurs in the sequence (Deutsch, 1975a, 1975b). This effect has been explained in terms of lateral inhibition, which results in the spreading of a memory distribution of the standard tone with respect not only to its pitch, but especially to its position in the sequence as time proceeds (Deutsch, 1984). Deutsch's studies have been applied to retroactive interference alone, although her theoretical formulation implies that interfering tones presented proactively, before a standard tone, should affect comparison performance as well. The same implication can be found in the trace discrimination hypothesis (Baddeley, 1976), which states that when two stimuli of the same class are presented and the last one is to be remembered, the ability to recall the last stimulus is dependent on how well it is distinguished temporally from the other. In line with this, pitch comparisons between tone pairs have been shown to be affected not only by intervals between the tones but also by their intervals from preceding pairs of tones (Cowan, Saults,

The author thanks Nelson Cowan, Diana Deutsch, David Lavond, Gilbert Mohr, John Wixted, and two anonymous reviewers for their comments on this manuscript; Michael Freeman, David Lavond, and Jan Wikgren for checking the language; and Asko Tolvanen for helping in statistical analyses. Correspondence concerning this article should be addressed to T. Ruusuvirta, Department of Psychology, University of Jyväskylä, P. O. Box 35, 40351 Jyväskylä, Finland (e-mail: ruusuvir@psyka.jyu.fi).
$\&$ Nugent, 1997), thus indicating the presence of proactive interference.

In the case of proactive interference, however, the underlying assumption that interfering tones in a sequence are involved individually is not self-evident. This argument is linked to neurophysiological studies demonstrating that compound representations of tone sequences can be stored in the auditory short-term memory (Näätänen, 1990; Saarinen, Paavilainen, Schröger, Tervaniemi, \& Näätänen, 1992; Sokolov, 1975). What is crucial regarding the present experiment is that such higher order representations enable stimuli that have not yet occurred in a sequence to be anticipated. This extrapolatory nature of the short-term memory was revealed when an illusory continuously melodically descending tone sequence (Shepard tones) was presented and occasionally interrupted by a tone consisting of the same pitch as the previous tone (Tervaniemi, Maury, \& Näätänen, 1994). These deviations elicited a deflection of an event-related potential, termed mismatch negativity (MMN), which is interpreted to reflect the preattentive detection of a stimulus change, a discrepancy between the current auditory input and the representation of invariant features of preceding stimuli in the short-term memory (for a review, see Näätänen, 1990). As each tone in the sequence was different in pitch, the representation could only be formed on the basis of the sequence as a compound (melodically descending series) and, thereby, was interpreted to represent an anticipation of the next stimulus in the sequence. In another study, MMN was similarly elicited when melodically ascending sequences of five tones separated by $1.02 \mathrm{sec}$ were presented with occasional fourth- or fifth-tone repetitions (Remijn \& Sugita, 1996). Interestingly, this interval is longer than the interval $(<200 \mathrm{msec})$ 


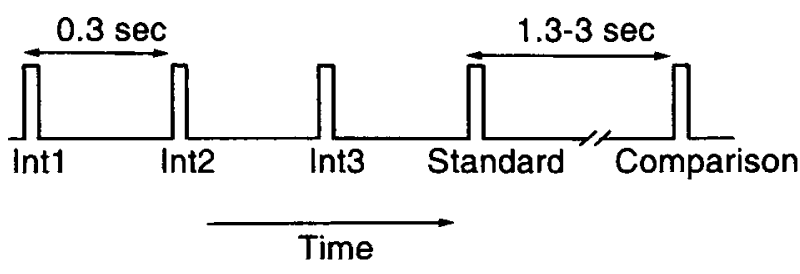

Figure 1. A schematic illustration of the stimulus arrangement. Int 1 to Int 3 refer to the interfering tones preceding the standard and comparison tones.

within which the auditory system is able to integrate sensory input into a single auditory percept (Tervaniemi, Saarinen, Paavilainen, Danilova, \& Näätänen, 1994; Warren \& Ackroff, 1976) and, in this respect, corresponds to that used in studies of retroactive interference in which a 300-msec interstimulus interval separated the interfering tones and a 2-sec delay separated the comparison tone from the last interfering tone (e.g., Deutsch, 1975a, 1975b).

The aim of the present study was to examine the extent to which a compound representation of a proactively presented sequence of interfering tones (melodic ordering of the tones) and their individual representations (pitches of individual tones) are involved in performance in a twotone pitch comparison task. For example, let us begin with three interfering tones that are lower in pitch than a standard tone and form a melodically ascending sequence ending in the standard tone that, in turn, precedes a comparison tone. Since the pitch of the comparison tone varies from trial to trial, being either below or above the pitch of the standard tone, different error patterns are to be expected, depending on whether the compound representation of the interfering tones or their individual representations are involved. If a compound representation is formed, the best comparison performance is to be expected when the pitch of the comparison tone is below the pitch of the standard tone. Owing to the interfering tones, the memorized pitch of the standard tone should be shifted toward a good continuation with the ascending sequence (Remijn \& Sugita, 1996; Tervaniemi, Maury, \& Näätänen, 1994) and, thereby, be in high contrast with the comparison tone. On the contrary, if the tones are individually represented, the best performance is to be expected when the pitch of the comparison tone is above the pitch of the standard tone. Then, spreading of a memory distribution concerning the position of the standard tone in the sequence should lead to high contrast between the memorized standard tone and the comparison tone, since the interfering and comparison tones deviate in pitch in opposite directions from the standard tone (Baddeley, 1976; Deutsch, 1984).

In the present study, different pitch combinations of interfering and comparison tones in relation to a standard tone are applied. From trial to trial, the pitch of the comparison tone varies, being either below or above the pitch of the standard tone. Also, the pitches of the interfering tones vary, being either below, above, or identical to the pitch of the standard tone. In addition, the interfering tones consisting of the pitches below and above the pitch of the standard tone are ordered either randomly or monotonically, analogous to the example above. If individual representations of the interfering tones are involved in the comparison performance, the error rate in performance should be the lowest when the interfering and comparison tones deviate in pitch in opposite directions from the standard tone, irrespective of the orderings. On the contrary, if a compound representation of the interfering tones is involved, the error rate should be the lowest when the interfering and comparison tones deviate in pitch in the same direction from the standard tone in the monotonically ordered trials. If both types of representations of the interfering tones are involved, the distribution of errors over different pitch combinations of the interfering and comparison tones should vary as a function of the orderings as well.

\section{METHOD}

\section{Subjects}

Fourteen university students with reported normal hearing served as subjects. None of the subjects possessed absolute pitch.

\section{Procedure}

A computer program was used in the data gathering. The subjects were presented with a sequence of tones consisting of three interfering tones followed by the standard tone and then by the comparison tone (Figure 1). They were instructed to ignore the interfering tones and to judge whether the pitch of the comparison tone was either below or above the pitch of the standard tone. The subjects were informed that the direction of deviation of the pitch of the comparison tone would be randomly selected for each trial. They responded by pressing the left or the right arrow key on the keyboard, depending on their judgments. The onset-to-onset interval between the three interfering tones and between the final interfering tone and the standard tone in a sequence was $300 \mathrm{msec}$. In turn, the separation between the standard and the comparison tones varied randomly between 1.3 and $3 \mathrm{sec}$. The interval between the subject's response and the next sequence was set randomly between 1.5 and $3 \mathrm{sec}$. Four hundred fifty trials were gathered from each subject.

\section{Stimuli}

All the sinusoidal $75-\mathrm{dB}$ tones were binaurally presented via earphones. They were $30 \mathrm{msec}$ in length, including $5-\mathrm{msec}$ rise/fall times, which has been shown to be sufficient for memory trace formation regarding pitch (Paavilainen, Jiang, Lavikainen, \& Näätänen, 1993). The frequencies of the interfering tones corresponded to the musical notes $G_{5}(783.99 \mathrm{~Hz}), A_{5}(880 \mathrm{~Hz}), B_{5}(987.77 \mathrm{~Hz})$, $\mathrm{C}_{6}(1046.5 \mathrm{~Hz}), \mathrm{D}_{6}(1174.7 \mathrm{~Hz}), \mathrm{E}_{6}(1318.5 \mathrm{~Hz})$, and $\mathrm{F}_{6}(1396.9 \mathrm{~Hz})$. A tone corresponding to the musical note $\mathrm{C}_{6}(1046.5 \mathrm{~Hz})$ was used as the standard tone. Tones deviating $\pm 7 \mathrm{~Hz}, \pm 14 \mathrm{~Hz}$, or $\pm 21 \mathrm{~Hz}$ from $\mathrm{C}_{6}(1025.5 \mathrm{~Hz}, 1032.5 \mathrm{~Hz}, 1039.5 \mathrm{~Hz}, 1053.5 \mathrm{~Hz}, 1060.5 \mathrm{~Hz}$, and $1067.5 \mathrm{~Hz}$ ) were used as the comparison tones.

\section{Interfering-tone sequences}

Sequences of three interfering tones were divided into those consisting of pitches below $\left(G_{5}, A_{5}, B_{5}\right)$, above $\left(D_{6}, E_{6}, F_{6}\right)$, or identical to that of the standard tone $\left(C_{6}\right)$. The sequences consisting of pitches above and below those of the standard tones were further divided into monotonic (melodically ascending if the pitches were below and descending if above the standard tone) and random or- 
Table 1

The Five Types of Interfering Sequences

Consisting of the Pitches Below, Above, or

Identical to the Pitch of the Standard Tone $\left(C_{6}\right)$

\begin{tabular}{|c|c|c|c|c|}
\hline \multicolumn{2}{|c|}{$\begin{array}{c}\text { Below } \\
\left(\mathrm{G}_{5}, \mathrm{~A}_{5}, \mathrm{~B}_{5}\right)\end{array}$} & \multirow{2}{*}{$\begin{array}{c}\begin{array}{c}\text { Identical } \\
\left(\mathrm{C}_{6}\right)\end{array} \\
\text { III } \\
\left(\mathrm{C}_{6}-\mathrm{C}_{6}-\mathrm{C}_{6}\right)\end{array}$} & \multicolumn{2}{|c|}{$\begin{array}{c}\text { Above } \\
\left(\mathrm{D}_{6}, \mathrm{E}_{6}, \mathrm{~F}_{6}\right)\end{array}$} \\
\hline $\begin{array}{c}I \\
\text { Ascending } \\
\left(G_{5}-A_{5}-B_{5}\right)\end{array}$ & $\begin{array}{c}\text { II } \\
\text { Random order }\end{array}$ & & $\begin{array}{c}\text { IV } \\
\text { Descending } \\
\left(\mathrm{F}_{6}-\mathrm{E}_{6}-\mathrm{D}_{6}\right)\end{array}$ & $\begin{array}{c}\mathrm{V} \\
\text { Random order }\end{array}$ \\
\hline
\end{tabular}

ders (Table 1). In the random assignment of the tones, monotonic sequences obtained by chance were excluded.

In each trial, the sequence type and the pitch of the comparison tone were randomly assigned.

\section{RESULTS}

The percentage of errors without interference (trials consisting of interfering tones identical to the standard tone) of the sample was 14.9. A repeated measures analysis of variance (ANOVA) was calculated, with the interference scales (the pitches of the interfering tones below, above, or identical to the pitch of the standard tone) and comparison tones (the pitch of the comparison tone below vs. above the pitch of the standard tone) as factors. The effect of either the interference scales or the comparison tones factor on the percentage of errors was not significant. However, their interaction was significant $[F(2,26)=95.12, p<.01 ; p$ value reported according to the Greenhouse-Geisser corrected degrees of freedom]. Thus, the percentage of errors depended on whether the comparison tone and the interfering tones deviated in pitch in the same or in the opposite direction from the standard tone (Figure 2). The error rate was higher when the interfering tones and the comparison tone deviated in pitch in the same direction from the standard tone than when the interfering tones and the standard tone were identical; it was lower when they deviated in opposite directions. This was confirmed by a repeated measures ANOVA $[F(1,13)=101.15, p<.01 ; p$ value reported according to the Greenhouse-Geisser corrected degrees of freedom], with one factor consisting of three levels (the interfering tones and the comparison tone deviating in pitch in the same direction from the standard tone, the interfering tones and the comparison tone deviating in pitch in opposite directions from the standard tone, and the interfering tones being identical in pitch to the standard tone), together with Bonferroni corrected post hoc pairwise comparisons, all three being statistically significant $(p<.001)$.

To reveal whether the random versus monotonic orderings of the interfering tones affected the distribution of the errors over the combinations of directions in which the interfering tones and the comparison tone deviated in pitch from the standard tone, a repeated measures ANOVA was calculated. It consisted of monotonicity (monotonically vs. randomly ordered interfering tones), interference scales (the pitches of the interfering tones below vs. above the pitch of the standard tone), and comparison tones (the pitch of the comparison tone below vs. above the pitch of the standard tone) as factors (Table 2). As in the first analysis in this section, the interaction between the interference scales and the comparison tones factors was significant $[F(1,13)=175.94, p<.001]$. No main effect of monotonicity was found. Furthermore, the interaction between the three factors was not significant $[F(1,13)=0.47, p=.55]$.

\section{DISCUSSION}

Interfering tones presented proactively affected the pitch comparisons made between standard and comparison tones. When the interfering and comparison tones deviated in pitch in opposite directions from the standard tone, the lowest error rate in performance was found. In turn, deviations in the same direction resulted in the highest error rate. An intermediate error rate was found when no interference was provided-that is, when the interfering tones were identical in pitch to the standard tone. Thus, the representation of the standard tone seemed to become intertwined with the interfering tones as constituents of the sequence, resulting in a bias in the memorized pitch of the standard tone toward their pitches, as would be expected on the basis of the lateral inhibition

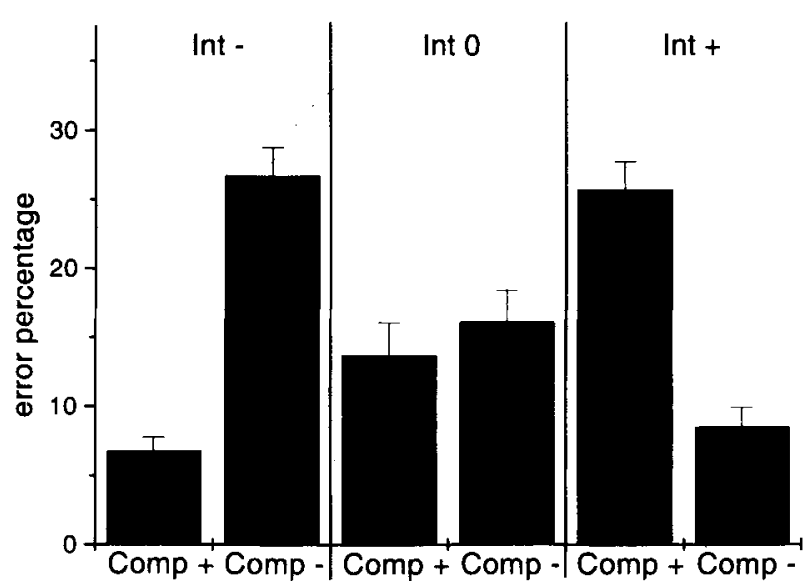

Figure 2. Error percentages in trials consisting of interfering tones of lower (Int - ), same (Int0), or higher (Int + ) pitch and comparison tones of lower (Comp-) or higher (Comp+) pitch than the standard tone. Error bars correspond to standard error. 
Table 2

Means (With Standard Errors) of Percentages of Errors

in Trials With Different Pitch Relationships Between the

Interfering and the Comparison Tones When the Interfering

Tones Are Monotonically Versus Randomly Ordered

\begin{tabular}{|c|c|c|c|c|c|c|c|c|}
\hline \multirow[b]{2}{*}{ Order } & \multicolumn{2}{|c|}{$\begin{array}{c}\text { Interfering + } \\
\text { Comparison - }\end{array}$} & \multicolumn{2}{|c|}{$\begin{array}{l}\text { Interfering - } \\
\text { Comparison }+ \\
\end{array}$} & \multicolumn{2}{|c|}{$\begin{array}{l}\text { Interfering - } \\
\text { Comparison - }\end{array}$} & \multicolumn{2}{|c|}{$\begin{array}{l}\text { Interfering + } \\
\text { Comparison + }\end{array}$} \\
\hline & $M$ & $S E$ & $M$ & $S E$ & $M$ & $S E$ & $M$ & $S E$ \\
\hline Monotonic & 7.6 & 1.62 & 7.8 & 1.33 & 26.2 & 1.94 & 28.1 & 1.95 \\
\hline Random & 9.4 & 1.64 & 5.6 & 1.09 & 27.2 & 2.70 & 23.3 & 2.32 \\
\hline
\end{tabular}

Note -+ refers to higher and - to lower pitch than the standard tone.

(Deutsch, 1984) and trace discrimination (Baddeley, 1976) hypotheses.

The orderings of the interfering tones (monotonic vs. random) were not found to affect the error patterns. Irrespective of the tone orderings, a higher error rate occurred when the interfering and comparison tones deviated in pitch in the same direction from the standard tone than when they deviated in opposite directions. Thus, there is no evidence that the memorized pitch of the standard tone became shifted toward a good continuation with the melodically ascending/descending interfering tone sequence in the monotonically ordered trials (Remijn \& Sugita, 1996; Tervaniemi, Maury, \& Näätänen, 1994). This observation agrees further with the lateral inhibition (Deutsch, 1984) and temporal discrimination (Baddeley, 1976) hypotheses, which focus on individual interfering tones in their effect on pitch comparisons. Again, the error rates seemed to merely reflect a confusion between memorized stimulus positions in the sequence.

Although I found no evidence of an effect of melodically ordered tones, as compared with randomly ordered tones, it is conceivable that such an effect existed but was too small to be detected in my experiment. Also, randomly ordered trials consisted of a melodically ascending or descending interval between the last interfering tone and the standard tone. This could have obscured the contrast between randomly and monotonically ordered sequences. However, these intervals were always preceded by earlier ones that were not in accordance with the monotonic ordering and thereby violated the coherence of the compound. It could also be suggested that the number of the interfering tones (four when the standard tone is taken into account) was too low for representations of melodic orderings of tones to be built into the memory, not to mention that studies on retroactive interference have used six or more interfering tones (Deutsch, 1975a, 1975b; see also Pechmann \& Mohr, 1992). However, pairs of tones have been found to be sufficient for representations of such orderings to be stored in the acoustic short-term memory (Saarinen et al., 1992).

Nevertheless, the possibility remains that both the compound representation of the interfering tones and their individual representations influenced performance on the present task. However, at the least, the effect of the latter would have to have been much stronger than the former. Again, it must be emphasized that if the distribution of er- rors over the combinations of the directions in which the interfering tones and the comparison tone deviated in pitch from the standard tone had been strongly affected by the compound representation, this distribution would have been precisely the opposite to what was observed in the monotonically ordered trials. That is, an error rate in performance would have been lower when the interfering and comparison tones deviated in pitch in the same direction from the standard tone than when they deviated in opposite directions.

In this respect, it is possible that the stimulus representation in the acoustic short-term memory that contributes to the preattentive detection of changes in homogenous auditory stimuli (Näätänen, 1990) may not be regarded as an analogue of the representation in the working memory (Baddeley \& Hitch, 1974) where conscious processes take place. Further support for such a dissociation can be found in a study in which the short-term memory, as revealed by MMN, was observed to store representations of auditory stimuli according to their physical and not their perceptual features (Ross, Tervaniemi, \& Näätänen, 1996). Further studies are clearly needed to settle these issues. They could also utilize the application of simple nonspeech stimulus attributes, since the auditory short-term memory has been shown to store pitches of speech sounds in a way similar to that for nonspeech sounds (Semal, Demany, Ueda, \& Hallé, 1996).

\section{REFERENCES}

BADDELEY, A. D. (1976). The psychology of memory. New York: Basic Books.

BADDEley, A. D., \& Hitch, G. (1974). Working memory. In G. H. Bower (Ed.), The psychology of learning and motivation (Vol. 8, pp. 47-89). New York: Academic Press.

Cowan, N., Saults, J. S., \& Nugent, L. D. (1997). The role of absolute and relative amounts of time in forgetting within immediate memory: The case of tone-pitch comparisons. Psychonomic Bulletin \& Review, 4, 393-397.

DEUTSCH, D. (1975a). Facilitation by repetition in recognition memory for tonal pitch. Memory \& Cognition, 3, 263-266.

DeursCH, D. (1975b). The organization of short-term memory for a single acoustic attribute. In D. Deutsch \& J. A. Deutsch (Eds.), Shortterm memory (pp. 107-151). New York: Academic Press.

DeutsCH, D. (1984). Memory for nonverbal auditory information: A link between behavioral and physiological studies. In L. R. Squire \& N. Butters (Eds.), Neuropsychology of memory (pp. 45-54). New York: Guilford.

NÄÄTÄNEN, R. (1990). The role of attention in auditory information processing as revealed by event-related potentials and other brain 
measures of cognitive function. Behavioral \& Brain Sciences, 13, 201-288.

Paavilainen, P., Jiang, D., Lavikainen, J., \& Nä̈̈tänen, R. (1993) Stimulus duration and the sensory memory trace: An event-related potential study. Biological Psychology, 35, 139-152.

PechmanN, T., \& Mohr, G. (1992). Interference in memory for tonal pitch: Implications for a working-memory model. Memory \& Cognition, 20, 314-320.

Remisn, G. B., \& SugitA, Y. (1996). Mismatch between anticipated and actually presented sound stimuli in humans. Neuroscience Letters, 202, 169-172.

Ross, J., Tervaniemi, M., \& Nä̈̈̈Ä̈nen, R. (1996). Neural mechanisms of the octave illusion: Electrophysiological evidence for central origin. NeuroReport, 8, 303-306.

Saarinen, J., Paavilainen, P., Schröger, E., Tervaniemi, M., \& NÄÄTÄNEN, R. (1992). Representation of abstract attributes of auditory stimuli in the human brain. NeuroReport, 3, 1149-1151.

Semal, C., Demany, L., Ueda, K., \& Hallé, P.-A. (1996). Speech ver- sus nonspeech in pitch memory. Journal of the Acoustical Society of America, 100, 1132-1140.

Sokolov, E. N. (1975). The neuronal mechanisms of the orienting reflex. In E. N. Sokolov \& O. S. Vinogradova (Eds.), Neuronal mechanisms of the orienting reflex (pp. 217-235). Hillsdale, NJ: Erlbaum. Tervaniemi, M, Maury, S., \& NäÄtÄnen, R. (1994). Neural representations of abstract stimulus features in the human brain as reflected by the mismatch negativity. NeuroReport, 5, 844-846.

Tervaniemi, M., Saarinen, J., Paavilainen, P., Danilova, N., \& NÄÄTÄNEN, R. (1994). Temporal integration of auditory information in sensory memory as reflected by mismatch negativity. Biological Psychology, 38, 157-167.

WARREN, R. M., \& ACKrofF, J. M. (1976). Two types of auditory sequence perception. Perception \& Psychophysics, 20, 387-394.

(Manuscript received January 9, 1998;

revision accepted for publication September 10, 1999.) 